Hadron Structure '15

International Journal of Modern Physics: Conference Series

Vol. 39 (2015) 1560094 (12 pages)

(C) The Author(s)

DOI: $10.1142 /$ S2010194515600940

\title{
Searches for exotic phenomena with the ATLAS detector
}

\author{
Patrick Czodrowski \\ (on behalf of the ATLAS Collaboration) \\ Department of Physics, University of Alberta \\ Edmonton, Alberta, Canada, T6G 2E1 \\ Patrick.Czodrowski@cern.ch
}

Published 26 November 2015

\begin{abstract}
Recent results of searches for exotic phenomena (new phenomena other than Supersymmetry) with the ATLAS experiment at the Large Hadron Collider conducted with proton-proton collision data at $\sqrt{s}=8 \mathrm{TeV}$ taken 2012 were presented.
\end{abstract}

Keywords: ATLAS; exotic; searches.

\section{Introduction}

Recent results of searches for exotic phenomena (new phenomena other than Supersymmetry) with the ATLAS experiment ${ }^{1}$ at the Large Hadron Collider (LHC) conducted with proton-proton collision data at a centre-of-mass energy of $\sqrt{s}=8 \mathrm{TeV}$ taken 2012 were presented. If not explicitly stated otherwise a dataset with $20.3 \mathrm{fb}^{-1}$ is used by the discussed analyses.

\section{Multi-Jet Search ${ }^{2}$}

A search for evidence of physics beyond the Standard Model (SM) in final states with multiple high-transverse-momentum jets is performed. To identify high- $p_{\mathrm{T}}$, high-multiplicity final states resulting from high-mass objects, a suitable variable is the scalar sum of the $p_{\mathrm{T}}$ of the jets in the event, $H_{\mathrm{T}}$. No significant excess of events beyond SM expectations is observed (see Fig. 1). A wide variety of models for black hole and string ball production and decay are considered, and the upper limit on the cross-section times acceptance is as low as $0.16 \mathrm{fb}$ at the $95 \%$ confidence level (CL) (see Fig. 2).

This is an Open Access article published by World Scientific Publishing Company. It is distributed under the terms of the Creative Commons Attribution 3.0 (CC-BY) License. Further distribution of this work is permitted, provided the original work is properly cited. 


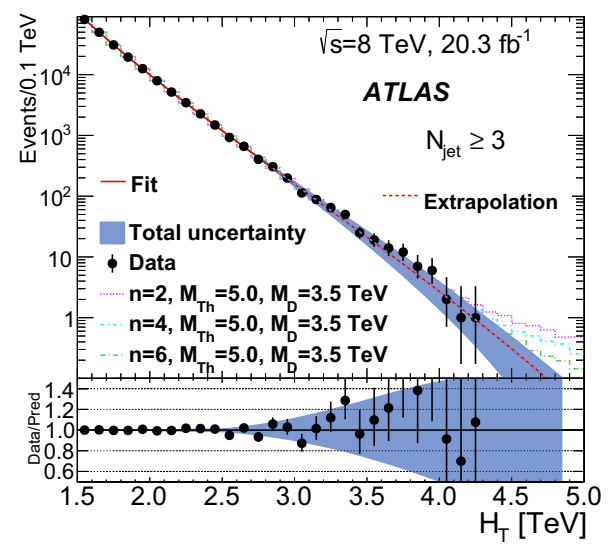

(a)

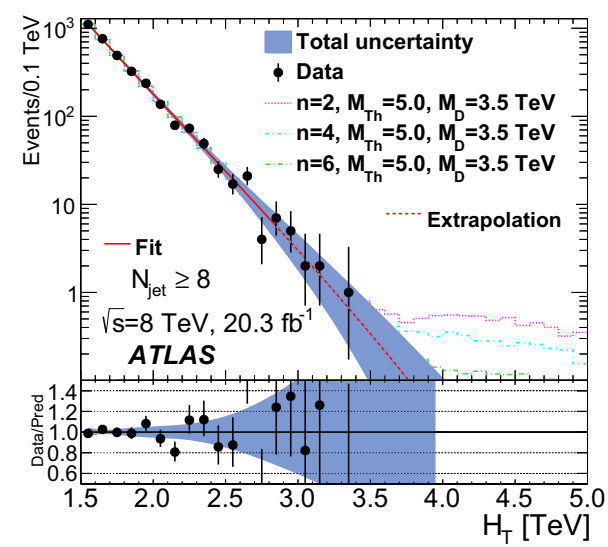

(b)

Fig. 1. The $H_{\mathrm{T}}$ distributions, showing the data and extrapolated fits from the control region $1.5<H_{\mathrm{T}}<2.9 \mathrm{TeV}$ into the signal region $H_{\mathrm{T}}>3.0$ for different jet multiplicities (a) $N_{\text {jet }} \geq 3$ and (b) $N_{\text {jet }} \geq 8$. The uncertainty on the data points in both the distribution and ratio are due to the statistical uncertainty on the data only. The uncertainty band includes all uncertainties on the background prediction. Also shown are the expected black hole signals for three parameter sets of the CHARYBDIS $2^{3}$ non-rotating black hole model. ${ }^{2}$

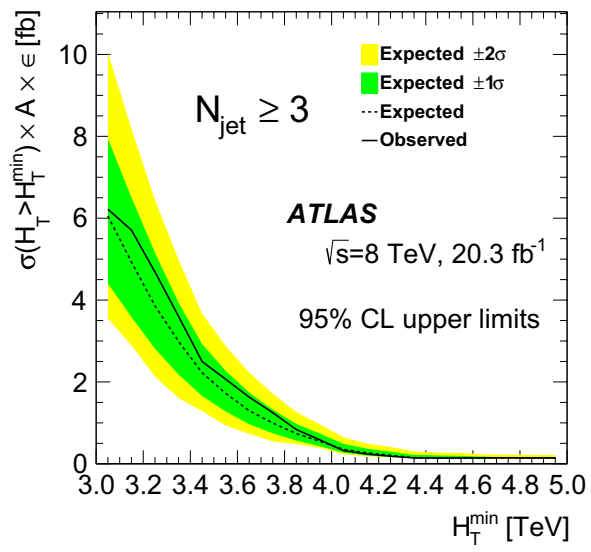

(a)

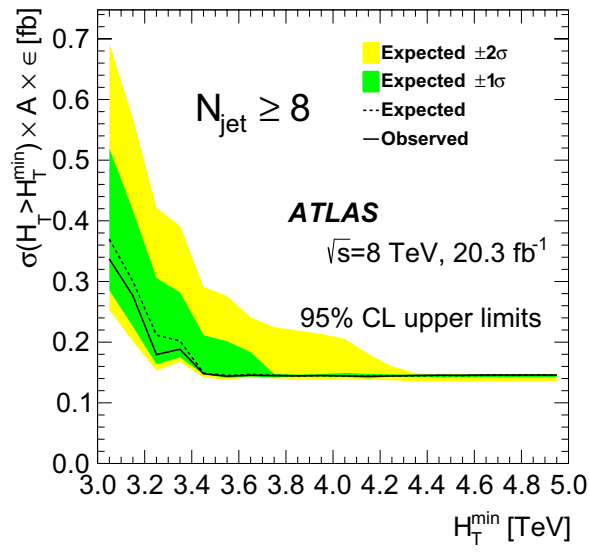

(b)

Fig. 2. Upper limits on the visible cross-section (cross-section $(\sigma)$ times acceptance $(A)$ times efficiency $(\epsilon))$ at the $95 \% \mathrm{CL}$ versus inclusive $H_{\mathrm{T}}$ for different inclusive jet multiplicities, (a) $N_{\text {jet }} \geq 3$ and (b) $N_{\text {jet }} \geq 8$. The solid (dashed) lines correspond to the observed (expected) upper limits. The green (dark) and yellow (light) bands represent one and two standard deviations from the expected limits. ${ }^{2}$ 


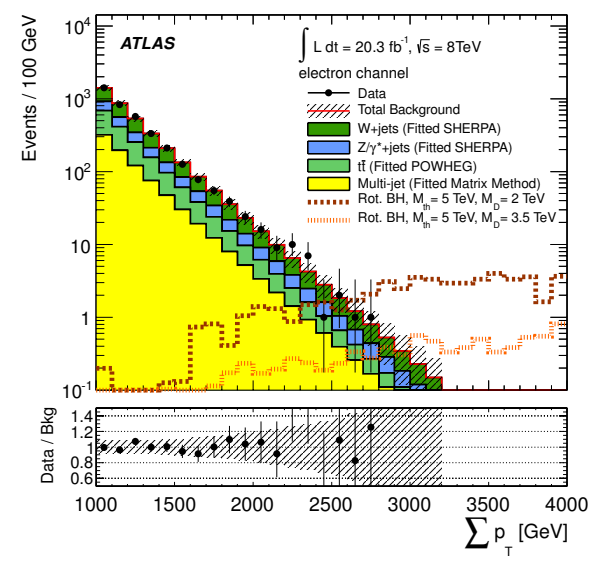

(a)

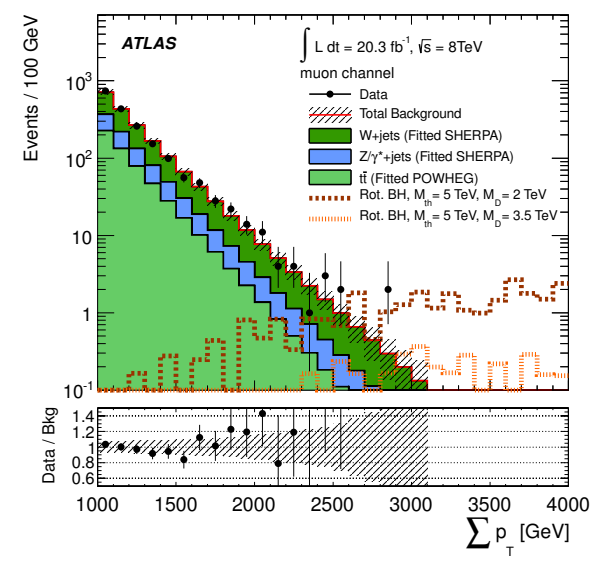

(b)

Fig. 3. The $\sum p_{\mathrm{T}}$ distributions in the (a) electron and (b) muon channels. Two representative signal distributions for rotating black holes with $n=6$ are overlaid to illustrate the signal properties. The lower panels show the ratio of the data to the expected background, with the statistical uncertainty on data (points), and separately, the fractional total uncertainty on the background (shaded band). ${ }^{4}$

\section{Search for Black Holes and String Balls with Leptons and Jets ${ }^{4}$}

A search for an excess of events with multiple high transverse momentum objects including charged leptons and jets is presented. No excess of events beyond SM expectations is observed (see Fig. 3). The analysis considers events at high $\sum p_{\mathrm{T}}$, defined as the scalar sum of the $p_{\mathrm{T}}$ of the selected reconstructed objects (hadronic jets and leptons), containing at least three high- $p_{\mathrm{T}}$ objects (leptons or jets), at least one of which must be a lepton. Using extra-dimensional models for black hole and string ball production and decay, exclusion contours are determined as a function of the mass threshold for production and the fundamental gravity scale for two, four and six extra dimensions (see Fig. 4). For six extra dimensions, mass thresholds of 4.8-6.2 TeV are excluded at 95\% CL, depending on the fundamental gravity scale and model assumptions.

\section{Dark Matter 6}

Results of a search for new phenomena in events with large missing transverse momentum and a Higgs boson decaying to two photons are reported. The observed data are well described by the expected SM backgrounds. Exclusion limits are presented for models of physics beyond the SM featuring dark matter candidates, as for example illustrated in Fig. 5. Prior to these results, no bounds have been placed by collider experiments on the Higgs boson + dark matter models. 


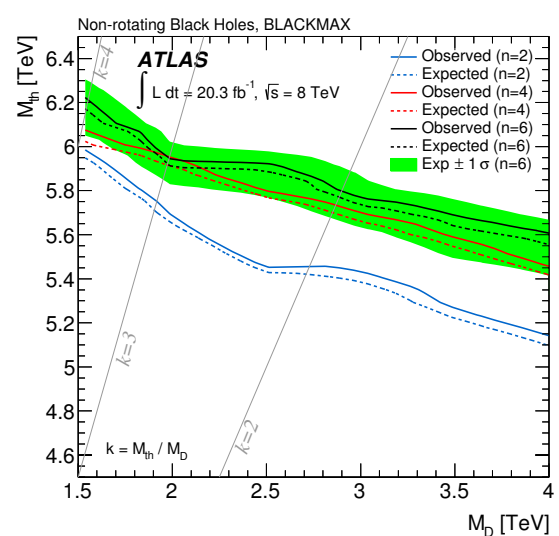

(a)

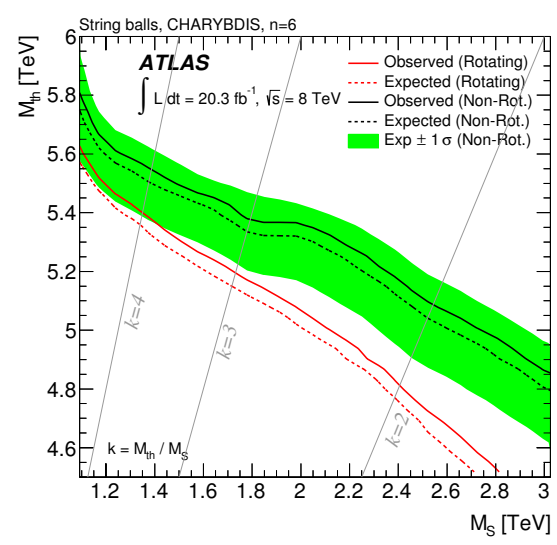

(b)

Fig. 4. The exclusion limits in the (a) $M_{\mathrm{th}}-M_{D}$ and (b) $M_{\mathrm{th}}-M_{\mathrm{S}}$ plane, with electron and muon channels combined, for (a) non-rotating black hole models two, four and six extra dimensions, simulated with BlackMax ${ }^{5}$ and (b) rotating and non-rotating string ball models with six extra dimensions. The solid (dashed) lines show the observed (expected) 95\% CL limits, with the band illustrating the expected $\pm 1 \sigma$ variation of the expected limits for the (a) $n=6$ (b) non-rotating case. The $\pm 1 \sigma$ variation is comparable for the (a) $n=2$ and $n=4$ models (b) rotating case. Masses below the corresponding lines are excluded. The lighter grey lines indicate constant (a) $k=M_{\mathrm{th}} / M_{D}$ (b) $k=M_{\mathrm{th}} / M_{\mathrm{S}} \cdot{ }^{4}$

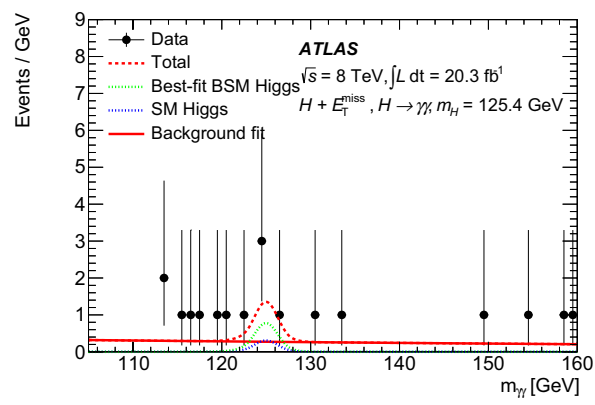

(a)

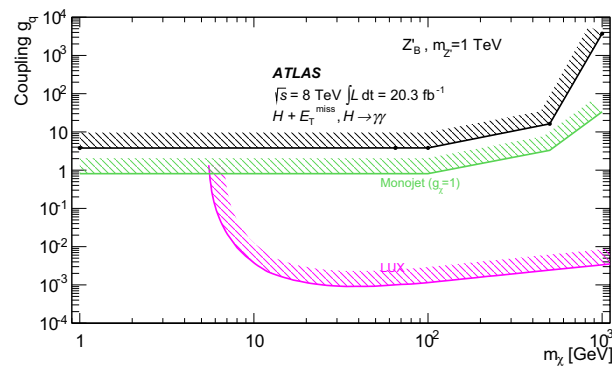

(b)

Fig. 5. (a) Distribution of the diphoton invariant mass $m_{\gamma \gamma}$. An unbinned maximum-likelihood fit to the spectrum is used to estimate the number of events from the continuum background and from $H \rightarrow \gamma \gamma$ decays the individual components are shown as well as their sum. (b) Limits on coupling parameters for simplified models with a heavy mediator with mass of $1 \mathrm{TeV}$. All constraint contours exclude larger couplings or mixing angles. Regions excluded due to perturbativity arguments are indicated red, green and pink contours denote results from collider searches for invisible $H$ decays, ${ }^{7}$ and monojet ${ }^{8}$ searches, and the LUX Collaboration, ${ }^{9}$ respectively. ${ }^{6}$ 


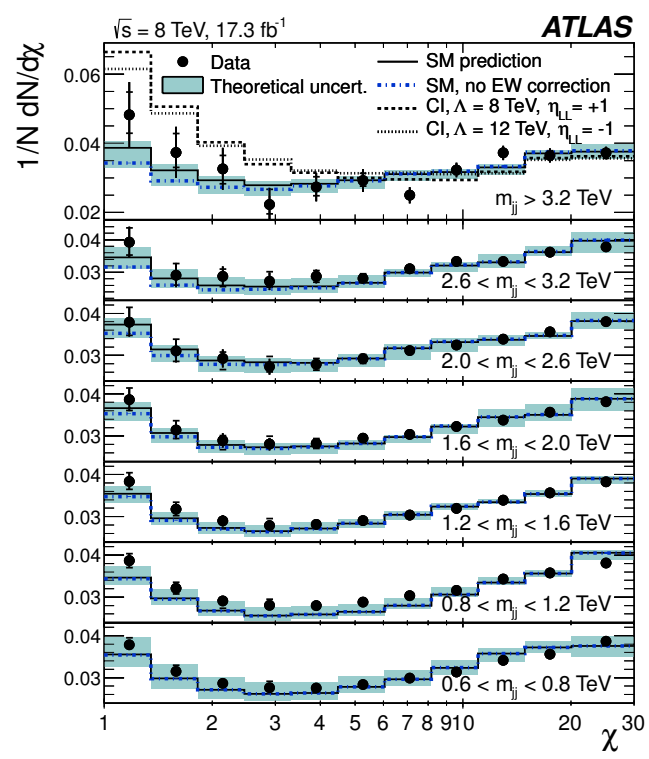

Fig. 6. Distributions of the dijet angular variable $\chi$ for several regions of dijet invariant mass $m_{j j}$. Shown are the data (dots), SM predictions (solid lines) with uncertainties, and predictions for a benchmark theory of new contact interactions with a left-chiral color-singlet coupling with constructive (dotted, $\eta_{\mathrm{LL}}=-1$ and $\Lambda=12 \mathrm{TeV}$ ) or destructive (dashed, $\eta_{\mathrm{LL}}=+1$ and $\Lambda=8 \mathrm{TeV}$ ) interference with the SM processes. The error bars on data represent the experimental and statistical uncertainties added in quadrature, with a tick representing experimental uncertainties only. The theoretical uncertainties are displayed as a shaded band around the SM prediction. ${ }^{10}$

\section{Excited Quarks and Quark Compositeness Searches with Dijet Angular Distributions ${ }^{10}$}

A search for new phenomena in LHC proton-proton collisions at $\sqrt{s}=8 \mathrm{TeV}$ was performed with the ATLAS detector using an integrated luminosity of $17.3 \mathrm{fb}^{-1}$. The angular distributions are studied in events with at least two jets the highest dijet mass observed is $5.5 \mathrm{TeV}$. All angular distributions are consistent with the predictions of the SM (see Fig. 6). In a benchmark model of quark contact interactions, a compositeness scale below $8.1 \mathrm{TeV}$ in a destructive interference scenario and $12.0 \mathrm{TeV}$ in a constructive interference scenario is excluded at 95\% CL.

\section{One Measurement Many Interpretations: Search for Top Quark Pair Resonances ${ }^{11}$}

A search for new particles that decay into top quark pairs, $t \bar{t}$, is reported. The lepton-plus-jets final state is used, where the top pair decays to $W^{+} b W^{-} \bar{b}$, with one $W$ boson decaying leptonically and the other hadronically. The invariant mass spectrum of top quark pairs is examined for local excesses or deficits that are inconsistent with the SM predictions (see Fig. 7). Events are reconstructed assuming the final state originated from a $t \bar{t}$ decay. To calculate $m_{t \bar{t}}^{\text {reco }}$, the neutrino four-momentum 


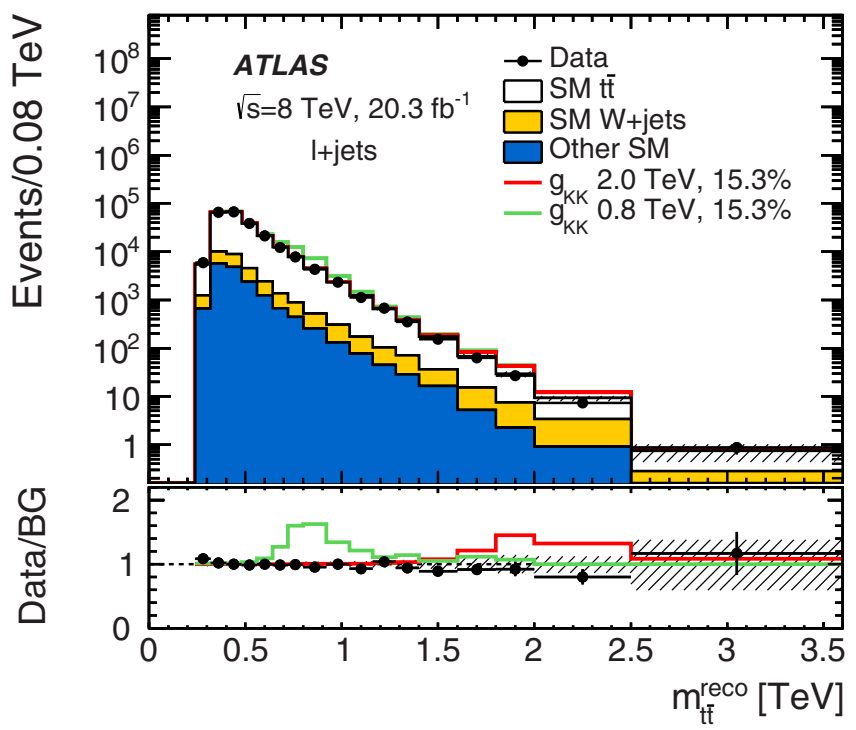

Fig. 7. The $m_{t \bar{t}}^{\text {reco }}$ distributions, after the nuisance-parameter fit under the background-only hypothesis, summed over all 12 channels compared with data. The SM background components are shown as stacked histograms. The shaded areas indicate the total systematic uncertainties. The red (green) line shows the expected distribution for a hypothetical $g_{\mathrm{KK}}$ of mass $2.0(0.8) \mathrm{TeV}$, width $15.3 \%{ }^{11}$

must be determined. The neutrino transverse momentum is taken to be the $E_{\mathrm{T}}^{\text {miss }}$ vector. The longitudinal component of the neutrino momentum, $p_{z}$, is calculated by constraining the lepton plus missing momentum system to have the $W$ boson mass and solving the resulting quadratic equation in the neutrino's longitudinal momentum $p_{z} \cdot{ }^{12,13}$ If no real solution exists, the $E_{\mathrm{T}}^{\mathrm{miss}}$ vector is varied by the minimal amount required to produce exactly one real solution. If two real solutions are found, the one with the smallest $\left|p_{z}\right|$ is used for the boosted-topology reconstruction, while the choice is made by a $\chi^{2}$ algorithm. For the boosted topology, $m_{t \bar{t}}^{\text {reco }}$ is computed from the four-momenta of the neutrino, lepton, the selected small-radius jet and the large-radius jet. In this case the assignment of jets to the semileptonically decaying top quark and hadronically decaying top quark is unambiguous. No evidence for a top quark pair resonance is found, and 95\% CL limits on the production rate are determined for massive states in benchmark models. The upper limits on the cross-section times branching ratio of a narrow a $Z^{\prime}$ boson decaying to top pairs range from $4.2 \mathrm{pb}$ to $0.03 \mathrm{pb}$ for resonance masses from $0.4 \mathrm{TeV}$ to $3.0 \mathrm{TeV}$. A narrow leptophobic topcolour a $Z^{\prime}$ boson with mass below $1.8 \mathrm{TeV}$ is excluded. Upper limits are set on the cross-section times branching ratio for a broad colour-octet resonance with $\Gamma / m=15 \%$ decaying to $t \bar{t}$. These range from $4.8 \mathrm{pb}$ to $0.09 \mathrm{pb}$ for masses from $0.4 \mathrm{TeV}$ to $3.0 \mathrm{TeV}$. A Kaluza-Klein excitation of the gluon in a Randall-Sundrum model is excluded for masses below $2.2 \mathrm{TeV}$ (see Fig. 8). 


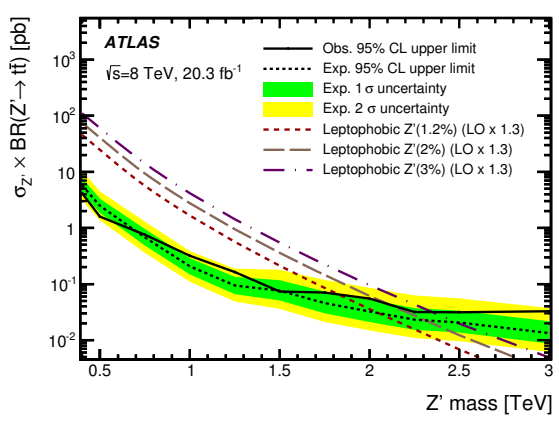

(a) $Z^{\prime}$, resolved and boosted combination.

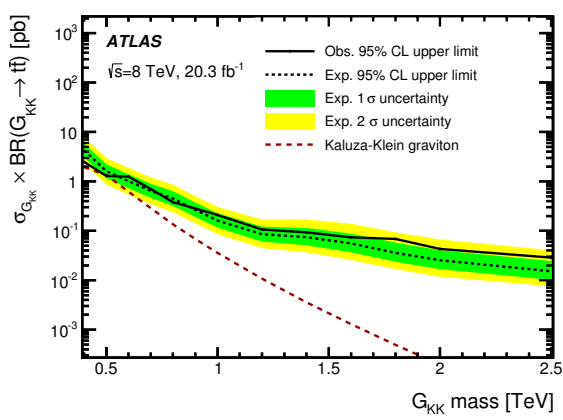

(c) $G_{\mathrm{KK}}$, resolved and boosted combination.

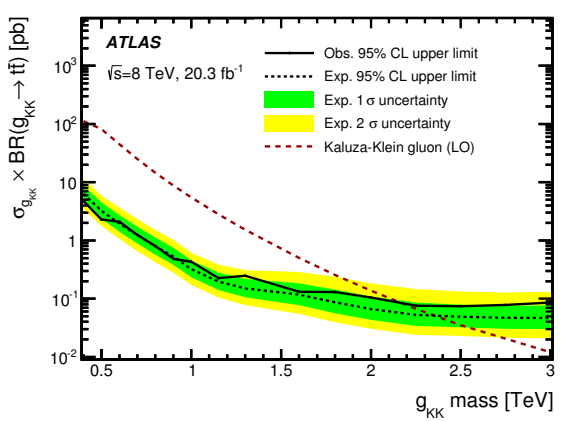

(b) $g_{\mathrm{KK}}$, resolved and boosted combination.

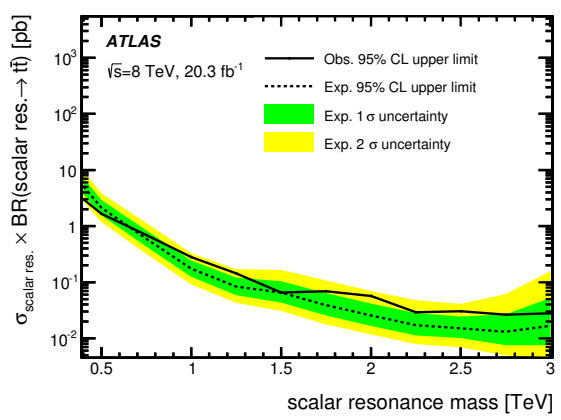

(d) Scalar resonance, resolved and boosted combination.

Fig. 8. Observed and expected upper limits on the production cross-section times branching ratio to $t \bar{t}$ final states as a function of the mass of (a) Topcolour-assisted-technicolour $Z_{\mathrm{TC} 2}^{\prime}$, (b) Bulk RS Kaluza-Klein gluon, (c) Bulk RS Kaluza-Klein graviton, (d) scalar resonance. The expected limits are derived from nominal (pre-fit) background estimates. The theoretical predictions for the production cross-section times branching ratio at the corresponding masses are also shown. ${ }^{11}$

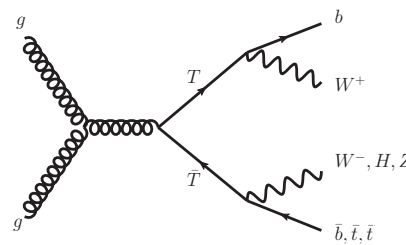

(a)

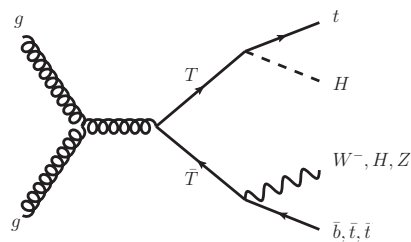

(b)

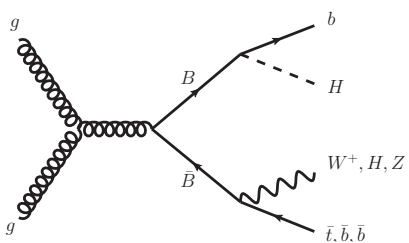

(c)

Fig. 9. Representative leading-order Feynman diagrams for $T \bar{T}$ production probed by (a) the $T \bar{T} \rightarrow W b+\mathrm{X}$ search and (b) the $T \bar{T} \rightarrow H t+\mathrm{X}$ search, and (c) for $B \bar{B}$ production probed by the $B \bar{B} \rightarrow H b+\mathrm{X}$ search. ${ }^{14}$ 


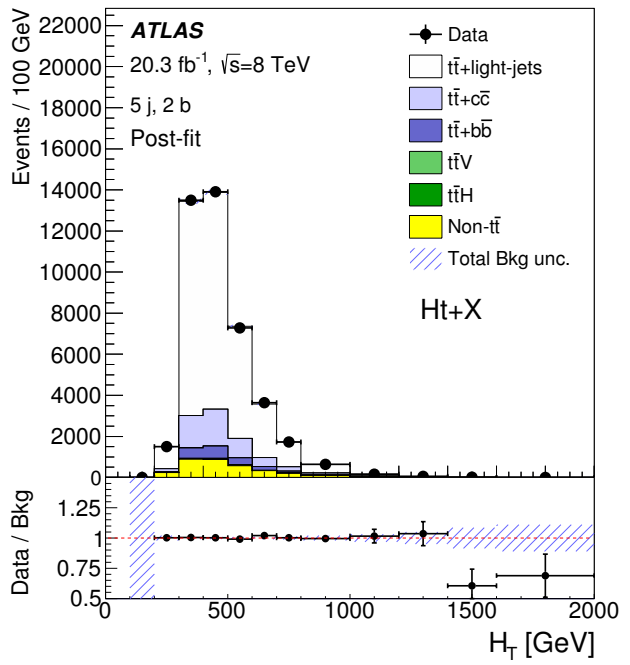

(a)

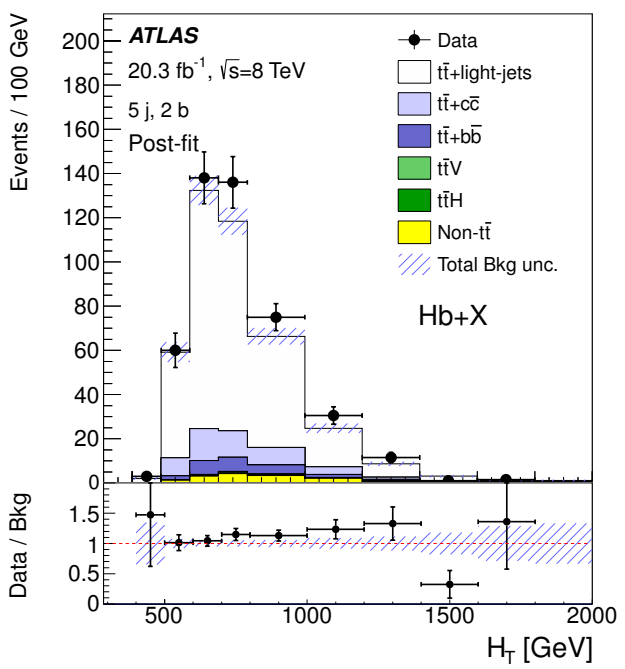

(c)

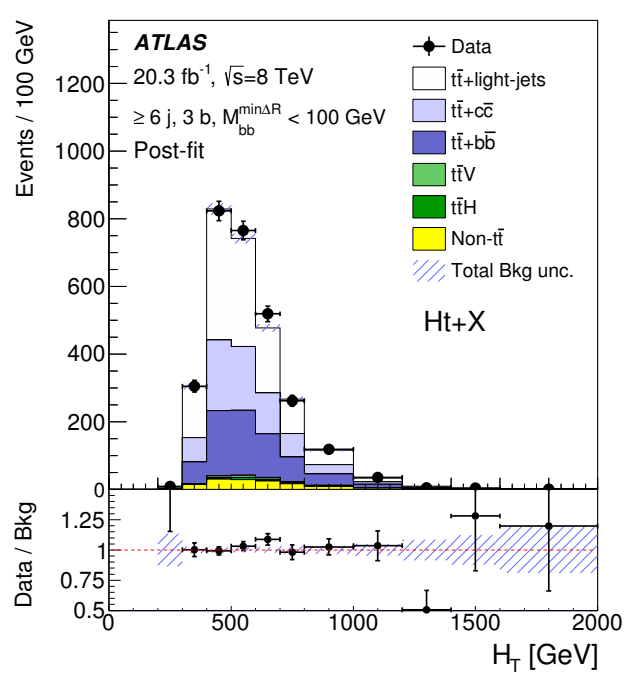

(b)

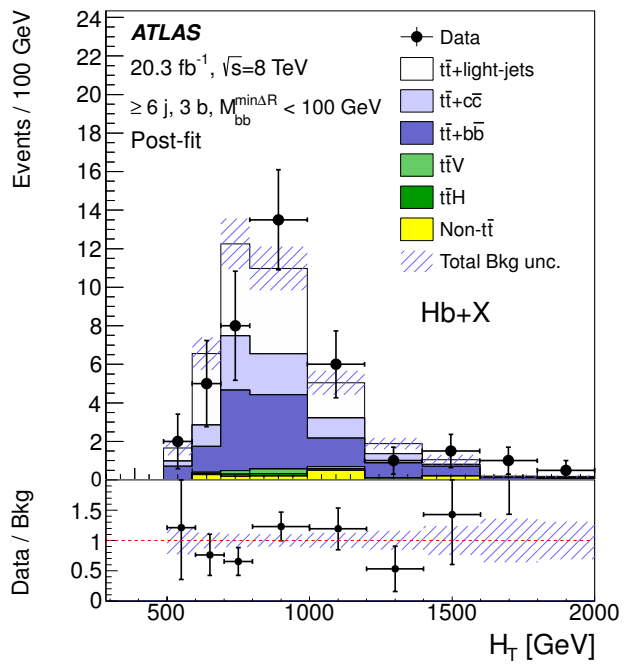

(d)

Fig. 10. $T \bar{T} \rightarrow H t+\mathrm{X}$ search: comparison between data and prediction for the distribution of the scalar sum $\left(H_{\mathrm{T}}\right)$ of the transverse momenta of the lepton, the selected jets and the missing transverse momentum in each of the analysed channels after final selection: (a) (5 j, $2 \mathrm{~b}),(\mathrm{b})(\geq 6$ $\mathrm{j}, 3 \mathrm{~b}$, low $\left.M_{b b}^{\min \Delta R}\right)$, (c) $(5 \mathrm{j}, 2 \mathrm{~b})$, and (d) $\left(\geq 6 \mathrm{j}, 3 \mathrm{~b}\right.$, low $\left.M_{b b}^{\min \Delta R}\right)$. The background prediction is shown after the fit to data under the background-only hypothesis. The small contributions from $W / Z+$ jets, single top, diboson and multi-jet backgrounds are combined into a single background source referred to as "Non- $t \bar{t}$ ". The last bin in all figures contains the overflow. The bottom panel displays the ratio of data to the total background prediction. The hashed area represents the total uncertainty on the background. ${ }^{14}$ 


\section{Vector Like Quarks ${ }^{14}$}

A search for pair production of vector-like quarks, both up-type $(T)$ and down-type $(B)$, as well as for four-top-quark production (see Fig. 9), is presented. Data are analysed in the lepton-plus-jets final state, characterised by an isolated electron or muon with high transverse momentum, large missing transverse momentum and multiple jets. Dedicated analyses are performed targeting three cases: a $T$ quark with significant branching ratio to a $W$ boson and a $b$-quark $(T \bar{T} \rightarrow W b+\mathrm{X})$, and both a $T$ quark and a $B$ quark with significant branching ratio to a Higgs boson and a third-generation quark $(T \bar{T} \rightarrow H t+\mathrm{X}$ and $B \bar{B} \rightarrow H b+\mathrm{X}$ respectively, see for example: Fig. 10). No significant excess of events above the SM expectation is observed, and 95\% CL lower limits are derived on the masses of the vector-like $T$ and $B$ quarks under several branching ratio hypotheses assuming contributions from $T \rightarrow W b, Z t, H t$ and $B \rightarrow W t, Z b, H b$ decays. The $95 \% \mathrm{CL}$ observed lower limits on the $T$ quark mass range between $715 \mathrm{GeV}$ and $950 \mathrm{GeV}$ for all possible values of the branching ratios into the three decay modes, and are the most stringent constraints to date (see Fig. 11).

\section{Heavy Long-Lived Multi-Charged Particles ${ }^{15}$}

A search for heavy long-lived multi-charged particles was presented. Particles producing anomalously high ionisation, consistent with long-lived massive particles with electric charges from $|q|=2 e$ to $|q|=6 e$ are searched for. No signal candidate events are observed, and 95\% CL cross-section upper limits are interpreted as lower mass limits for a Drell-Yan production model (see: Fig. 12). The mass limits range between 660 and $785 \mathrm{GeV}$.

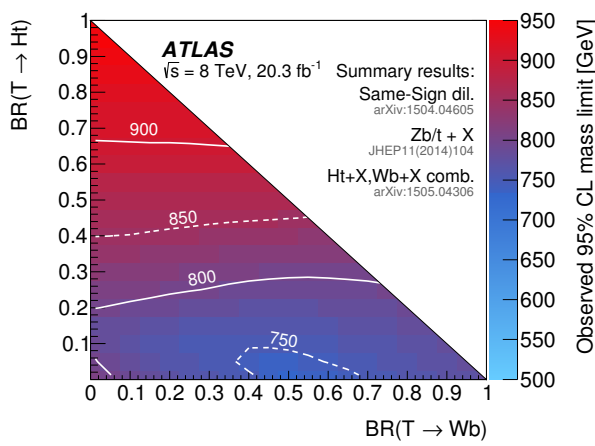

(a)

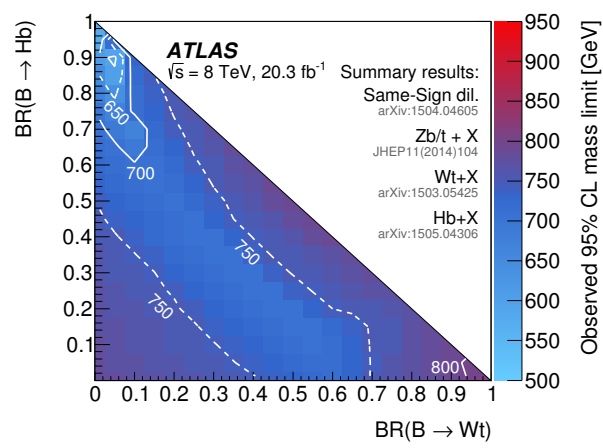

(b)

Fig. 11. Summary of the most restrictive observed limit (95\% CL) on the mass of the (a) $T$ quark in the plane of $\mathrm{BR}(T \rightarrow H t)$ versus $\mathrm{BR}(T \rightarrow W b)$ and (b) $B$ quark in the plane of $\operatorname{BR}(B \rightarrow H b)$ versus $\mathrm{BR}(B \rightarrow W t)$ from all ATLAS searches for $T \bar{T}$ or $B \bar{B}$ production, respectively. Contour lines are provided to guide the eye. ${ }^{14}$ 


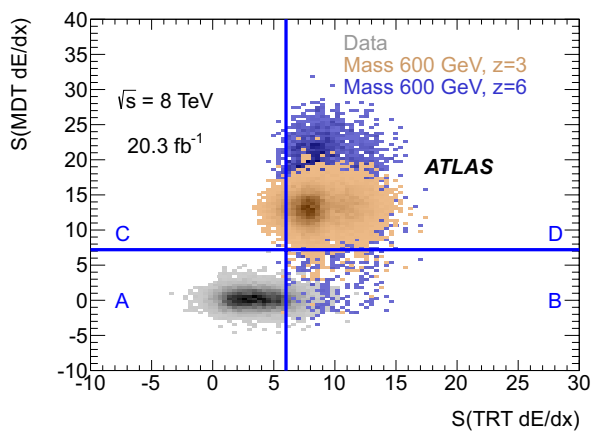

(a)

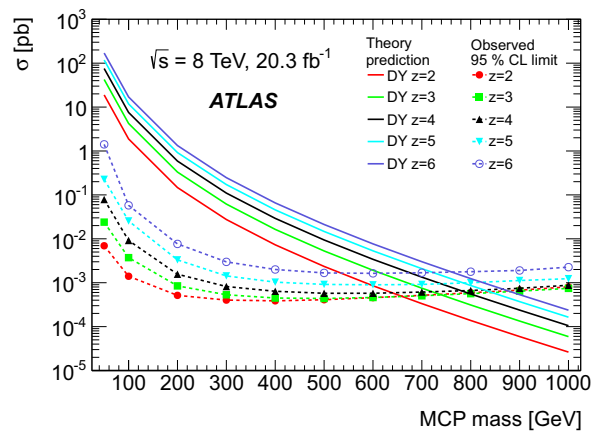

(b)

Fig. 12. (a) Normalised distributions of the $\mathrm{d} E / \mathrm{d} x$ significance in the Monitored Drift Tubes (MDT), $S($ MDT $\mathrm{d} E / \mathrm{d} x)$ versus the Transition Radiation Tracker of the Inner Detector (TRT), $S$ (TRT $\mathrm{d} E / \mathrm{d} x$ ) after the $z \geq 3$ tight selection. The distributions of the data and the simulated signal samples (here for a mass of $600 \mathrm{GeV}$ ) are shown. (b) Observed 95\% CL cross-section upper limits and theoretical cross-sections as functions of the multi-charged particle's mass for values of $z$ between 2 and $6 .^{15}$

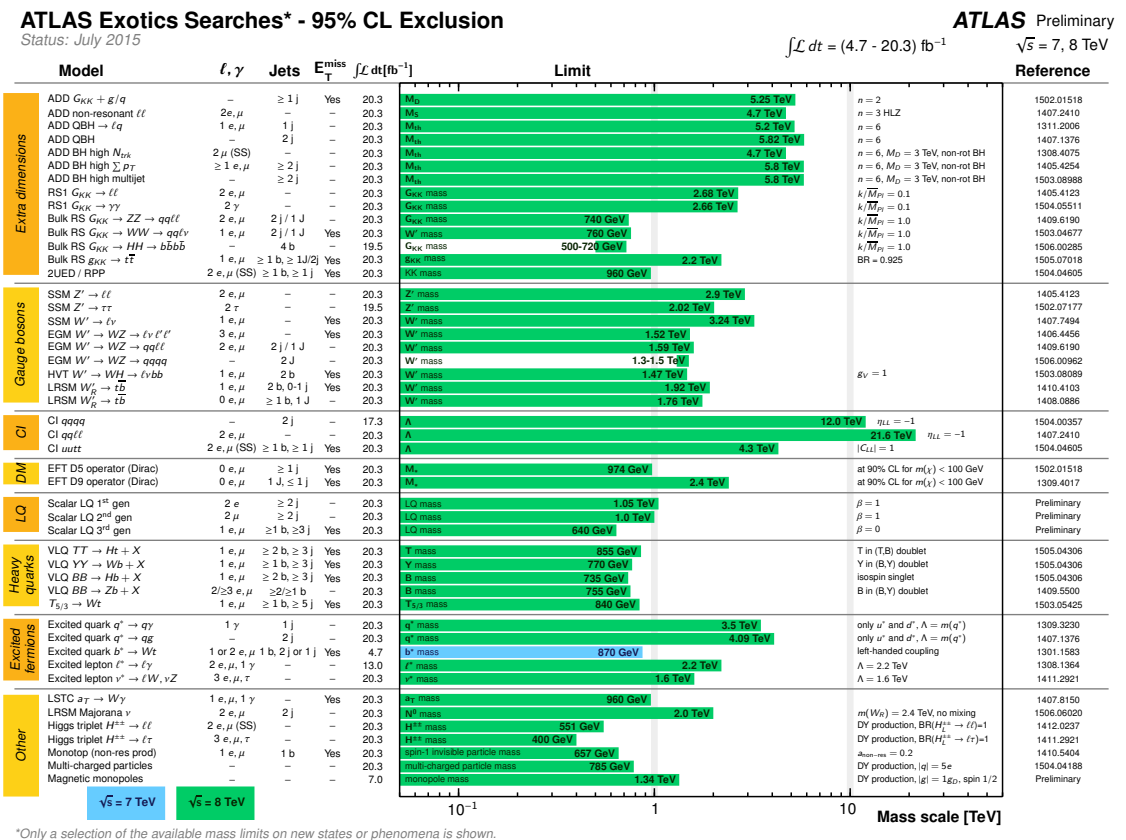

Fig. 13. Reach of ATLAS searches for new phenomena other than Supersymmetry. Blue (green) bands indicate $7 \mathrm{TeV}(8 \mathrm{TeV})$ data results. Only a representative selection of the available results is shown (always up-to-date results are available here: https://twiki.cern.ch/twiki/bin/view/ AtlasPublic/ExoticsPublicResults). 


\section{Outlook}

Searches for exotic signatures with the ATLAS detector were presented. A nonexhaustive overview of results of the ATLAS Exotics group is provided in Fig. 13. In the LHC Run II data and its analyses the cross-section improvements due to the increased centre-of-mass energy of the LHC of $\sqrt{s}=13 \mathrm{TeV}$ compared to $\sqrt{s}=8 \mathrm{TeV}$ in Run I will significantly expand our sensitivity for new physics.

\section{Acknowledgments}

We thank CERN for the very successful operation of the LHC, as well as the support staff from our institutions without whom ATLAS could not be operated efficiently.

We acknowledge the support of ANPCyT, Argentina; YerPhI, Armenia; ARC, Australia; BMWFW and FWF, Austria; ANAS, Azerbaijan; SSTC, Belarus; CNPq and FAPESP, Brazil; NSERC, NRC and CFI, Canada; CERN; CONICYT, Chile; CAS, MOST and NSFC, China; COLCIENCIAS, Colombia; MSMT CR, MPO CR and VSC CR, Czech Republic; DNRF, DNSRC and Lundbeck Foundation, Denmark; EPLANET, ERC and NSRF, European Union; IN2P3-CNRS, CEADSM/IRFU, France; GNSF, Georgia; BMBF, DFG, HGF, MPG and AvH Foundation, Germany; GSRT and NSRF, Greece; RGC, Hong Kong SAR, China; ISF, MINERVA, GIF, I-CORE and Benoziyo Center, Israel; INFN, Italy; MEXT and JSPS, Japan; CNRST, Morocco; FOM and NWO, Netherlands; BRF and RCN, Norway; MNiSW and NCN, Poland; GRICES and FCT, Portugal; MNE/IFA, Romania; MES of Russia and NRC KI, Russian Federation; JINR; MSTD, Serbia; MSSR, Slovakia; ARRS and MIZŠ, Slovenia; DST/NRF, South Africa; MINECO, Spain; SRC and Wallenberg Foundation, Sweden; SER, SNSF and Cantons of Bern and Geneva, Switzerland; NSC, Taiwan; TAEK, Turkey; STFC, the Royal Society and Leverhulme Trust, United Kingdom; DOE and NSF, United States of America.

The crucial computing support from all WLCG partners is acknowledged gratefully, in particular from CERN and the ATLAS Tier-1 facilities at TRIUMF (Canada), NDGF (Denmark, Norway, Sweden), CC-IN2P3 (France), KIT/GridKA (Germany), INFN-CNAF (Italy), NL-T1 (Netherlands), PIC (Spain), ASGC (Taiwan), RAL (UK) and BNL (USA) and in the Tier-2 facilities worldwide.

\section{References}

1. ATLAS Collaboration, JINST 03, S08003 (2008).

2. ATLAS Collaboration, JHEP 07, 032 (2015).

3. J. A. Frost et al., JHEP 10, 014 (2009).

4. ATLAS Collaboration, JHEP 08, 103 (2014).

5. D.-C. Dai et al., Phys. Rev. D77, 076007 (2008).

6. ATLAS Collaboration, CERN-PH-EP-2015-120 (2015), arXiv:1506.01081 [hep-ex].

7. G. Belanger, B. Dumont, U. Ellwanger, J. F. Gunion and S. Kraml, Phys. Rev. D88, 075008 (2013).

8. ATLAS Collaboration, Phys. Lett. B705, 294 (2011).

9. D. S. Akerib et al., Phys. Rev. Lett. 112, 091303 (2014). 
10. ATLAS Collaboration, Phys. Rev. Lett. 114, 221802 (2015).

11. ATLAS Collaboration, CERN-PH-EP-2015-090 (2015), arXiv:1505.07018 [hep-ex].

12. T. Aaltonen et al., Phys. Rev. D82, 112005 (2010).

13. ATLAS Collaboration, Phys. Lett. B717, 330 (2012).

14. ATLAS Collaboration, JHEP 08, 105 (2015).

15. ATLAS Collaboration, Eur. Phys. J. C75, 362 (2015). 